elevated serum lactic dehydrogenase level (1821 U/L); elevated $C$ reactive protein level $(15.3 \mathrm{mg} / \mathrm{dl})$; low thrombocyte count $\left(89-94 \times 10^{9} / \mathrm{L}\right)$; normal total and differential leukocyte counts; and normal prothrombin and partial thromboplastin time. Serological test for HIV was negative. Tumor markers including CA199, CA125, NSE, CEA, AFP were all within the normal range. Cerebrospinal fluid (CSF) opening pressure was $80 \mathrm{mmH}_{2} \mathrm{O}$, cell count was 450 cells $\times 10^{6} / \mathrm{L}$ and 10 leukocytes $\times 10^{6} / \mathrm{L}$, protein was $0.628 \mathrm{~g} / \mathrm{L}$ and sugar was normal. Cytology for malignant cells was negative.

Ultrasound of the abdomen was normal and computed tomography (CT) scan of chest and abdomen were normal. Non-contrast magnetic resonance imaging (MRI) scan demonstrated an abnormal low-signal area of $1.5 \times 2 \times 3 \mathrm{~cm}$ in the right frontal lobe. Contrast study showed nodular enhancement of the lesion with mild surrounding edema [Figure 1]. He had recurrence of seizures on day nine of admission and non-contrast MRI done on the day of third seizure revealed hemorrhage in the previous right frontal lobe and also a new hemorrhagic lesion in the left temporal lobe [Figure 2].

Patient was treated with antiepileptic medication and corticosteroids. He continued to have recurrent

\title{
Intravascular lymphomatosis with recurrent cerebral hemorrhages
}

\begin{abstract}
Sir,
Intravascular lymphomatosis (IL) is a rare lymphoproliferative disorder characterized by proliferation of neoplastic lymphocytes within vessel lumen with no or minimal parenchymal involvement. The central nervous system and skin are the most common sites, however, rarely other organ involvement has also been described. ${ }^{[1-6]}$ We report a patient with IL presenting with focal cerebral mass lesion and recurrent cerebral hemorrhage.
\end{abstract}

A 47-year-old male presented with two episodes of focal onset tonic-clonic seizures with secondary generalization in the one week before admission. On admission, general physical and neurological examination were normal, except for moderate anterograde amnesia. Laboratory investigation showed: Elevated erythrocyte sedimentation rate $(58 \mathrm{~mm} 1 \mathrm{~h})$;

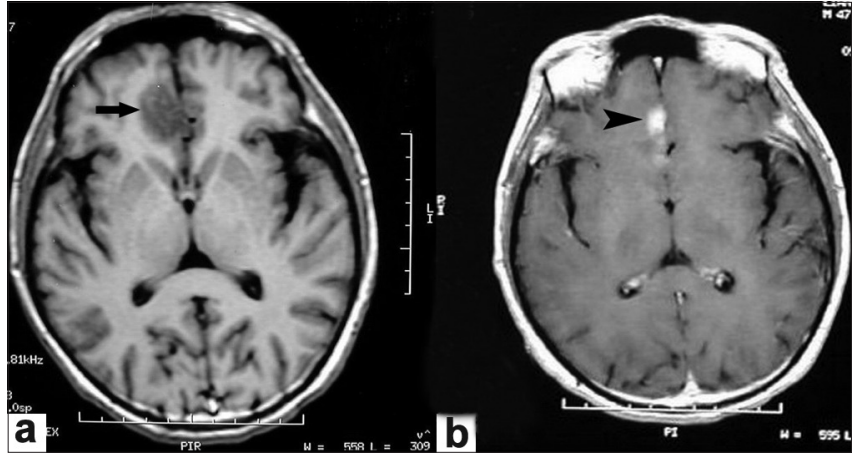

Figure 1: (a) On the same day of the second seizure plain MRI scan demonstrates an abnormal low-signal area of $1.5 \times 2 \times 3 \mathrm{~cm}$ in the right frontal lobe on T1 image (arrow). (b) Contrast MRI two days later showed nodular enhancement in the lesion, mild surrounding edema, the mass effect was not prominent (arrow head)

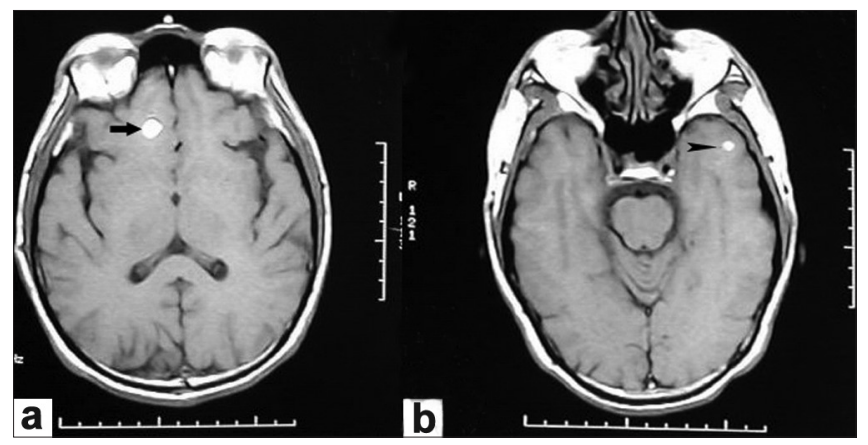

Figure $2(a, b)$ : Plain MRI scan nine days later showed hemorrhage at the lesion in the frontal lobe (arrow), also in the left temporal lobe (arrow head) 
seizures. Stereotactic brain biopsy of the right frontal lesion done one and half months of the presentation showed hemorrhage, gliosis, and calcification. During the follow-up he had repeated MRI and CT studies which revealed multiple hemorrhages [Figure 3]. Diagnostic possibility of agniitis was considered and cerebral angigram was done which was essentially normal. After three and half months of onset of the illness he developed fever, right sided weakness and progressive dementia and two weeks later he lapsed into coma. An emergent CT scan demonstrated a left hemispheric mass lesion with lateral ventricle effacement and midline shift, Emergent left subtemporal decompression was performed and the lesion was biopsied. Pathological examination of the lesion showed proliferation of large, atypical lymphoid cells in the lumen of small vessels and cell infiltration beyond the vessel wall into the adjacent brain parenchyma. Immunocytochemistry showed positive cells for CD20 confirming B-cell IL [Figure 4]. Bone marrow done fifteen days after the emergent brain operation showed neoplastic cells (single or

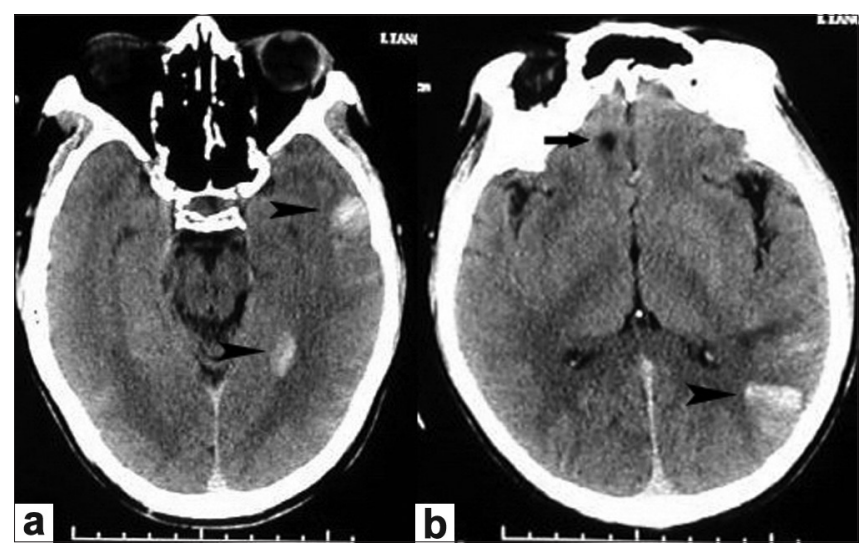

Figure $3(a, b)$ : One month later, plain CT scan showed new hemorrhage in the left hemisphere (arrow head), the low density in the right frontal lobe was the location of the biopsy (arrow)

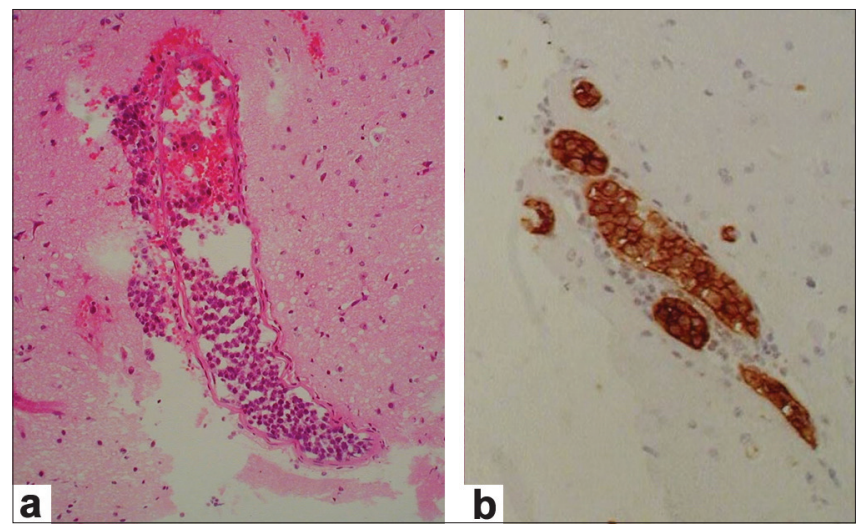

Figure 4: (a) Proliferation of large, atypical lymphoid cells in the lumen of small vessels; some cells infiltrated beyond the vessel wall into the adjacent neural tissue $(H$ and $E$, original magnification, $\times 100)$. (b) The immunocytochemistry studies showed these cells expressed B-cell markers $(C D 20)(\mathrm{IH}$, original magnification, $\times 200)$ clusters) with large round nucleus, fine chromatin, prominent nucleoli and basophilic cytoplasm with vacuolation. After the operation the patient was treated with five cycles of chemotherapy: Cyclophosphamide, adriamycin, vincristine, and prednisolone regimen (CHOP), followed by cranial radiotherapy. The mental status improved transiently however, aphasia, and hemiparesis remained unchanged. He continued to have recurrent seizures. He died 17 months after the presentation and autopsy could not be conducted.

Intravascular lymphomatosis is difficult to diagnose ante-mortem because of its variable clinical presentation and nonspecific laboratory findings. ${ }^{[7]}$ Neuroimaging findings are also nonspecific and include mass lesions, infacts with or without hemorrhagic transformation, and nonspecific white matter lesions. ${ }^{[8]}$ In our patient the initial MRI showed an enhancing mass lesion which later developed hemorrhage. Subsequent MRI and CT scans done during the course of illness showed recurrent hemorrhagic lesions with no perilesional edema. We feel that these hemorrhagic lesions were related to the primary pathology, IL. In IL, the tumor cells are predominantly or entirely within the vascular lumen however, extravascular extension may occur. In our patient, the pathological examination showed proliferation of large, atypical lymphoid cells in the lumen of small vessels, at the same time cell infiltration beyond the vessel wall into the adjacent brain parenchyma. The hemorrhagic lesions seen on the CT and MRI probably represent these lesions. The clinical presentation, course, and neuroimaging findings in our patient suggest that one should suspect the diagnostic possibility of IL with such presentation and a brain biopsy should be done to exclude IL.

\section{Cheng Xia, Sen-Yang Lang, Xue-Mei Li,} Sheng-Yuan Yu

Department of Neurology, General Hospital of PLA, China. E-mail: langsy1999@sina.com PMID: *** DOI: $10.4103 / 0028-3886.59492$

\section{References}

1. Cossu A, Deiana A, Lissia A, Satta A, Cossu M, Dedola MF, et al. Nephrotic syndrome and angiotropic lymphoma report of a case. Tumori 2004;90:510-3

2. Chim CS, Loong F. Intravascular lymphomatosis of the prostate gland. Br J Haematol 2002;119:2.

3. Lui PC, Wong GK, Poon WS, Tse GM. Intravascular lymphomatosis J Clin Pathol 2003;56:468-70.

4. Viali, S, Hutchinson DO, Hawkins TE, Croxson MC, Thomas M, Allen JP, et al. Presentation of intravascular lymphomatosis as lumbosacral polyradiculopathy. Muscle Nerve 2000;23:1295-300.

5. Walls JG, Hong YG, Cox JE, McCabe KM, O'Brien KE, Allerton JP, etal. Pulmonary intravascular lymphomatosis: Presentation with dyspnea and air trapping. Chest 1999;115:1207-10. 
6. Xabier B, Biagio A. The neurological masquerade of intravascular lymphomatosis. Arch Neurol 2002;59:439-43.

7. Liew CL, Shyu WC, Tsao WL, Li H. Intravascular lymphomatosis mimicks a cerebral demyelinating disorder. Acta Neurol Taiwan 2006;15:264-8.

8. Martin DN, Mokhtar K, Behin A, Lafitte F, Hoang-Xuan K, Chiras J. Intravascular malignant lymphomatosis. Neuroradiology 2002;44:749-54.

Accepted on 28-01-2009 